\title{
The Future of Infrared Spectroscopy in Biosciences: In Vitro, Time-Resolved, and 3D
}

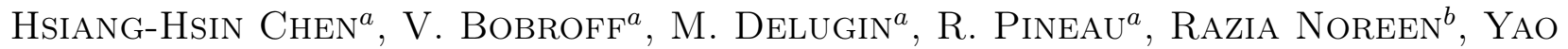

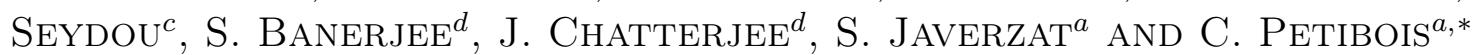 \\ ${ }^{a}$ Université de Bordeaux, Inserm U1029 LAMC, Allée Geoffroy Saint-Hillaire, Bat B2, 33600 Pessac, France \\ ${ }^{b}$ Department of Applied Chemistry and Biochemistry, Govt. College, University, Faisalabad, Pakistan \\ ${ }^{c}$ Centre for NanoHealth, Institute of Life Science 2 Building, Swansea University, Singleton Park, Swansea, \\ Abertawe, SA2 8PP, UK \\ ${ }^{d}$ School of Medical Science and Technology, Indian Institute of Technology, Kharagpur-721302, India
}

\begin{abstract}
Infrared (IR) spectroscopy is at the cross-roads, with the requirement to compete with cutting-edge technologies in biosciences, mostly based on analytical performances dealing with the super-resolutions: time, lateral/spatial, and contrast. IR microscopy is diffraction limited in most cases, thus not accessing to high lat$\mathrm{eral} / \mathrm{spatial}$ resolutions. Additionally, it has a poor signal-to-noise ratio on a single scan, thus requiring long-lasting acquisitions that are not suitable to analyze ns-lasting biochemical events. However, it is unique because it provides a broad global chemical information of the sample contents. It is also unique because it does not require heavy sample preparation nor labeling and can be coupled to other techniques (multimodality). Finally, it is again unique because it provides quantitative measurements, thus suitable for $1 \mathrm{D}$ to $4 \mathrm{D}$ data exploitation procedures. This short review shows that IR spectroscopy will be certainly subjected to a second century of innovations, maintaining its influence in the panorama of cutting-edge analytical techniques.
\end{abstract}

DOI: $10.12693 /$ APhysPolA.129.255

PACS: 87.10.Ca

\section{Introduction}

FTIR spectroscopy has been developed more than a century ago for analytical chemistry purposes, in 1893 in its principle by Nichols [1] and popularized a bit later by William Coblentz [2]. The purpose was to develop the first spectra database of molecules and to release tables of wavelengths at which various materials absorb IR light. For almost half a century [3], FTIR spectroscopy has remained a unique analytical resource to probe the structure of small molecules, but its influence in chemistry has been minored by the development of other techniques, notably nuclear magnetic resonance (NMR) and crystallography. After a century of instrumentation development, the main use of this technique has moved from molecular analyses towards the understanding of complex biological systems [4]. In the 90's, FTIR spectroscopy instrumentation focused on other applications with the release of spectrometers able to collect spectra on sample holders dedicated to quantitative, thus reproducible and comparable, measurements. The study of biofluids [5-7] has first shown that triplicate measurements allowed to obtain high reproducibility in spectra acquisitions with no more than $1 \%$ of change in absorptions on average $[8,9]$.

\footnotetext{
* corresponding author; e-mail cyril.petibois@u-bordeaux.fr
}

Another major advance in FTIR instrumentation for biological applications has been the development of the microscopes, as while their principle was established as early as 1949 [10], their ability to be used with both visible and IR modes [11] was found useful for bioscience. FTIR imaging systems have the advantage of a quite fast spectra acquisition since the microscope is coupled to an array of IR detectors, which may be linear (16 or 32 detectors) or focal plane $(64 \times 64$ or $128 \times 128$ detectors $)$ [11]. At this state of technological development, FTIR imaging was considered as cutting-edge technology for biosciences since the reduction of time for spectra acquisitions [12] allowed applications to large tissue areas and thus opened IR to the medical field [13]. The use of high-intensity (or high-photon flux) sources could be also considered as a major advantage to obtain high $\mathrm{S} / \mathrm{R}$ levels with shorter acquisition durations [14-17]. Cell imaging applications were also demonstrated taking into account a few analytical requirements to fit analytical standards in biology [14, 16, 18-20]. However, in the current competition for proposing high-performance analytical means for biosciences, it seems that FTIR instrumentations suffer from a major lack of R\&D efforts, and traditional manufacturers have not released major innovations for almost 20 years. It results that the most obvious advantages of FTIR spectroscopy and microscopy for biosciences a global chemical characterization of unaltered samples without labeling - are now completely outreached by many other techniques, notably those based on shorter wavelengths (UV to X-ray range). In this context, several key challenges must be considered by the IR spectroscopy 
community to open new avenues for IR applications in biosciences. This review tackles the main R\&D efforts to consent for achieving this objective.

\section{Analytical requirements for bioimaging by IR spectroscopy}

Molecular bio-imaging has imposed a new way to analyze cells and tissues (Fig. 1). From vibrational spectroscopies (mainly infrared, and Raman) to particle-based techniques (using energy of X-rays, $\gamma$-rays...), a wide range of microscopy techniques now fit some of the analytical features required for biological investigations [21]. The first feature is about the spatial resolution, which is determined by the physical principle of the technique [22]. In the electromagnetic spectrum, the wave-matter interactions can be exploited to reveal biosample features within the $\mathrm{nm}$ to $\mathrm{cm}$ range. The $\mathrm{nm}$ dimension refers to molecules, the fundamental bricks of living systems, and the $\mathrm{cm}$ range refers to the functional units (tissue structures) of these ones (Fig. 1). Because they are complex and structured systems, cells and tissues require to obtain data only at the micrometric scale or better for morphological analysis (anatomic imaging, from cytoskeleton in cells to multicellular assemblies in tissues). Major techniques exist for sub- $\mu \mathrm{m}$ analysis of cells and their subcompartments [19]. The place of IR microscopy is interesting in this panorama of analytical techniques as it allows analyzing some of the most fundamental structures of biological specimens, i.e. at $\mu \mathrm{m}$-scale dimensions, thus at the sub-cellular, cell, and sub-tissue levels. This is a range of dimensions that is currently poorly covered by existing microscopy techniques, which are either resolving super-resolution features on small volumes (or limited field-of-view), or do not provide sufficient lateral/spatial resolutions on larger specimens $\left(\mathrm{mm}^{3}\right.$ or bigger $)$. IR microscopy has the advantage of a quite low diffraction limit (typically $\approx 3-20 \mu \mathrm{m}$ for the $4000-500 \mathrm{~cm}^{-1}$ mid-IR interval), but also a weak penetration depth $(5-30 \mu \mathrm{m}$ for soft materials like tissues), thus limiting its applications in biosciences.

Another feature to consider in biological imaging is the temporal resolution, which will determine the applications of techniques for time-resolved analyses. This temporal resolution for biological applications is also related to sample stability during data acquisitions [23]. The physical properties of imaging modalities will determine their applicability on time-resolved analyses in biology. Most of imaging modalities using accelerator-based sources are not designed for analyzing living specimens due to the utilization of high energy beams. However, molecular dynamics may be determined out of the living environment, such as for the analysis of proteins folding or conformation under stress conditions $(\mathrm{pH}$, temperature, etc.), where high energy may be required for time-resolved data acquisitions within the ms-ns range of time [24].

The third main feature to consider for determining the applicability of imaging modalities to the biologi-

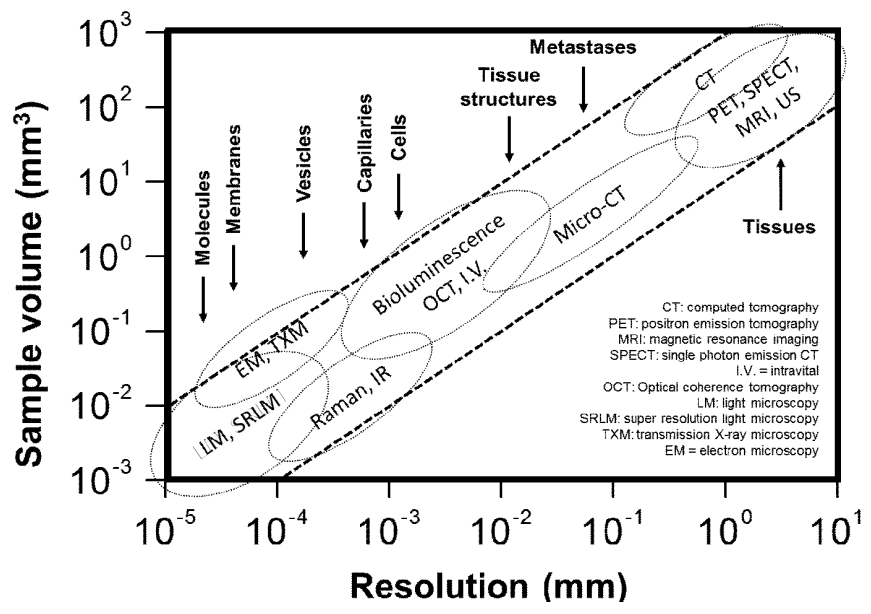

Fig. 1. Analytical techniques allowing a microscopic analysis of biosamples, from nanometer to millimeter dimensions and for different biological targets.

cal field is the sensitivity. In this domain, it is clear that accelerator-based sources play their most significant role for enhancing analytical performances, namely by increasing the $\mathrm{S} / \mathrm{N}$ level. This allows one to perform data acquisitions closer to the diffraction limit or to reduce the time of acquisition for obtaining information on kinetics of biological samples, the so-called time-resolved experiments. In this sense, IR microscopy has not been considered as a valuable technique for biological dynamics. The major developments of IR instrumentation to biological applications so far have been focused on sub-diffraction live cell imaging $[15,17,20]$ and high-throughput 2D tissue analyses $[11,18,21,25]$. Therefore, in the battle for achieving the best compromise between sensitivity, resolution, and time of acquisition, IR microscopy remains limited by the low-photon flux of its conventional IR sources (Glowbar) and the diffraction limit.

\section{From FTIR to IR spectroscopy and microscopy - the road to $3 \mathrm{D}$}

In principle, FTIR microscopy has been too slow in spectra acquisitions and provides too weak $\mathrm{S} / \mathrm{N}$ levels to allow comparison between large spectra data sets, i.e. millions to billions of spectra. The $\mathrm{S} / \mathrm{N}$ being dependent on environmental factors as well as electrical noise from the analytical instrumentation, there is little chance to obtain highly reproducible spectra for long-lasting acquisitions. This limit is affecting biological applications in the sense that quantitative changes in many chemical contents distribution (in cells or tissues) will remain very limited for physiological reasons. The example of glucose concentration in a normal physiological condition is a good demonstrator of what the biological variability is: blood concentration is normally ranging between 0.8 and $1.3 \mathrm{~g} / \mathrm{L}$ and remain very close to $1 \mathrm{~g} / \mathrm{L}$ in most of the cellular and extracellular compartments. Therefore, one may not expect major changes in glucose concentration 
distribution in tissues, and analyzing this molecular parameter at the microscopic spatial resolution will require accurate quantitative method with low margin of error in measurements, at least not superior to $2-5 \%$ of average value. FTIR spectroscopy and microscopy have not demonstrated that level of accuracy on triplicate measurements in biofluids [8,9], and thus one may not expect obtaining such analytical performance on successive tissue sections analysis. This slow acquisition issue must be overcome to expect developing FTIR microscopy for 3D applications. Although IR microscopy is not suitable for direct tomographic measurements on biosamples - because: 1 - IR photons have limited depth of penetration in matter [11, 14], 2 - biosamples contain $>60 \%$ of water with unmanageable absorptions [20], and 3 - transmission measurements do not allow resolving absorptions in a true confocal geometry [17] — its quantitative nature should allow the reconstruction of a 3D matrix of spectra.

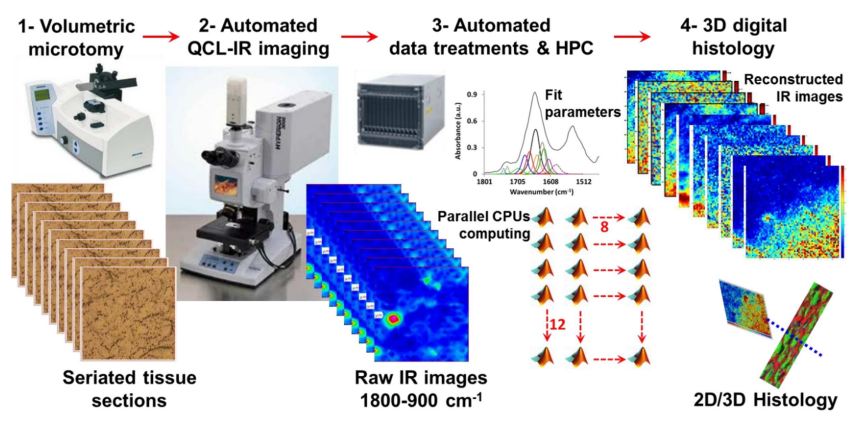

Fig. 2. Developing 3D IR microscopy for tissue block analysis. The concept of 3D digital histology can be developed by IR microscopy due to its intrinsic advantages, i.e., a global chemical analysis of biosample contents, a quantitative access to these ones, and a suitable sampling methodology for unlimited chemical data treatments of raw matter. The proposed methodology includes an automated tissue sectioning for obtaining reliable $2 \mathrm{D}$ images (visible and IR) that can be further stacked for 3D block reconstruction. Spectral data treatments are further performed for reconstructing 3D chemical information distribution with quantitative value in the tissue.

The recent release of microscopes powered by IR-laser sources is allowing new approaches for biological applications, with the opportunity to obtain high $\mathrm{S} / \mathrm{N}$ spectra in short time, probably 2 or 3 orders of magnitude faster than with the Glowbar sources. The wavelengthby-wavelength signal acquisition do not require the use of the Fourier transformation for spectra reconstruction (thus reducing mathematical approximations in absorption calculations), and provides absolute count of photons on detector, thus ensuring more accurate quantitative analysis of biosamples. These microscopes are not yet exempt from limits. They use several quantum-cascade lasers to cover a large spectral region (1700-900 $\mathrm{cm}^{-1}$ is currently proposed), which poses the question of the signal intensity reliability and continuity between lasers.
This is a fundamental question to consider quantitative analyses of chemical contents in 3D tissue blocks. Anyway, with a fast and high $\mathrm{S} / \mathrm{N}$ level spectra acquisitions, IR microscopy should allow reconstructing a large $3 \mathrm{D}$ matrix of IR spectra for tissue analyses (see proposed method in Fig. 2). The main purpose might be to introduce a new concept of spectral data treatments and exploitation, named "spectromics" [26], which is essentially the use of any spectral information (or combination or calculation of data extracted from spectra) that allows discrimination of two objects (e.g. healthy vs. pathological tissue or sub-structures inside the same tissue volume). Different spectral data treatment methods have been developed so far to extract an individual (e.g. an IR band) or a well-identified (e.g. amide I region) chemical information from spectra. The $3 \mathrm{D}$ reconstruction of these data might provide unique information about a tissue, with 3D resolved sub-structures correlated to biochemical or metabolic events. This would lead to the combination of morphological and molecular data into the same 3D image of a biosample, thus comparable to the MRI/CT or PET/CT multimodal imaging methods, but with three major advantages: 1 - a single technique would be used (thus using a single dataset of IR data), 2 - 3D reconstruction will be performed at the microscopic scale (down to $\lambda / 2$, i.e., a $3 \mu \mathrm{m}$ spatial resolution is achievable), and 3 - without the use of any label or imaging contrast agent, thus avoiding sample manipulations and alterations.

\section{From dry to wet biosampling in IR microscopy, and down to nanoscopy}

Recently, it has been proposed to use the attenuated total reflectance (ATR) acquisition method to obtain live cell images without constrain of the diffraction limit [20]. With synchrotron radiation source, this imaging can be performed in short time, i.e., in less than one minute, thus allowing to characterize macromolecular sub-cellular contents change [15]. Again, benefiting from the above mentioned innovations for high-speed and quantitative analyses, IR microscopy might become the first analytical technique able to determine metabolic and biochemical change in cells in vitro, thus opening the way to pharmacological experimentations on dose-response effects of drugs at the single cell level $[19,27]$. In fact, live cell imaging might concentrate all the benefits from technological innovations found in the new line of IR microscopes, with time-resolved, highly sensitive and quantitative measurement below the diffraction limit (down to $\lambda / 400$ as IR-laser sources are coherent, thus not limited by diffraction, which is reinforced by the use of ATR devices that are collecting the evanescent wave on surface of the reflecting substrate), all features collectively inaccessible for any other microscopy technique, except maybe for the Raman, but this one will be less sensitive and more resolutive than the IR microscopy. This might open again the debate between these two vibrational spec- 
troscopy techniques for high-end microscopy applications in biosciences, to the delight of spectroscopists!

The in vitro applications with ATR devices have been also developed for the measurement of nanoscaled biosamples [28], namely proteins or sub-cellular species, such as chromosomes of polymerized biomolecules [29]. The coupling to an atomic force microscope (AFM) is fundamental as it allows a $3 \mathrm{D}$ rendering that can be coupled to the co-localized acquisition of IR spectra, thus providing a volumetric and quantitative analysis of nanosized biosamples (Fig. 3); down to pixels of ten $\mathrm{nm}$ only [30]. This innovation should allow the development of fundamental experiments that are currently not possible with X-ray free electron lasers (X-FEL) or other super-resolution analytical techniques, namely the realtime nanoscopy in structural biology [31]. The example shown in Fig. 3 is showing possible application for in vitro analysis of membrane protein (a receptor) interaction with a ligand (here a growth factor), which can be performed with biochemical/metabolic cascade of event if a microfluidic device is developed for that purpose.

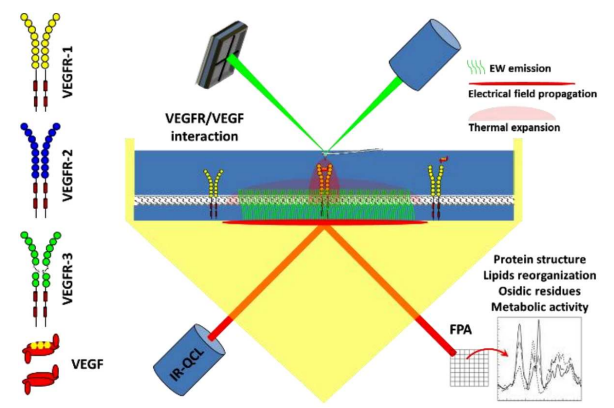

Fig. 3. Principle of time-resolved in vitroIR spectroscopy, with high-speed and nanometric analyses. Nano-IR analyses are feasible with 3D rendering from AFM measurements. The example here shows a sketch for protein-receptor (VEGF/VEGFR) interaction analysis by both non-contact liquid AFM and ATR-IR microscopies in a coordinated mode. A ms-range spectral acquisition is possible with analysis of conformational change sin protein-receptor interaction by IR spectroscopy using the amide I\&II spectral interval (1700-1500 $\mathrm{cm}^{-1}$ ) and with polarized beam for magnifying the amideI/amideII absorption changes.

\section{Conclusion}

IR spectroscopy offers significant analytical advantages over most of the other techniques in the field with a quantitative and global chemical rendering of a biosample. The recent methodological efforts allowing to perform in vitro analyses for single cell imaging and data treatments methods for extracting given chemical data from spectra, among other examples, show the relevance of the development of a new line of microscopes, much faster, sensitive and resolutive than the first generation. Laserpowered IR microscopes are arriving and promise analytical performances that should facilitate the development of time-resolved (in the ms range) and 3D (for tissues) applications with incomparable outputs. The strength of IR-lasers is also making that nanoresolved species can be analyzed at $\lambda / 400$, thus approaching the single molecule level with quantitative and volumetric rendering thanks to the coupling of an AFM. This should reintroduce the IR microscopy and nanoscopy into the panel of cutting-edge technologies for enhancing our knowledge on biosamples, from single molecule, to single cell and to tissues (even organs in small animal models), as well as in routine analyses for biomedical and biological research purposes.

\section{References}

[1] E.F. Nichols, Phys. Rev. 1, 1 (1893).

[2] W.W. Coblentz, Investigations of Infrared Spectra, in: T.P.P.N.M. USA, Carnegie Institute of Washington, Washington D.C. 1905, p. 1.

[3] E.J. Ambrose, A. Elliott, Proc. R. Soc. Lond. 206, 206 (1951).

[4] D. Naumann, D. Helm, H. Labischinski, Nature 351, 81 (1991).

[5] C. Petibois, V. Rigalleau, A.M. Melin, A. Perromat, G. Cazorla, H. Gin, G. Deleris, Clin. Chem. 45, 1530 (1999).

[6] G. Budinova, J. Salva, K. Volka, Appl. Spectrosc. 51 631 (1997).

[7] K.J. Ward, D.M. Haaland, M.R. Robinson, R.P. Eaton, Proc. SPIE 1145, 607 (1989).

[8] C. Petibois, G. Cazorla, A. Cassaigne, G. Deleris, Clin. Chem. 47, 730 (2001).

[9] C. Petibois, G. Cazorla, H. Gin, G. Deleris, J. Lab. Clin. Med. 137, 184 (2001).

[10] R. Barer, A.R.H. Cole, H.W. Thompson, Nature 163, 198 (1949).

[11] C. Petibois, G. Déléris, Trends Biotechnol. 24, 455 (2006).

[12] P. Innocenzi, T. Kidchob, J.M. Bertolo, M. Piccinini, M.C. Guidi, C. Marcelli, J. Phys. Chem. B 110, 10837 (2006).

[13] D.C. Fernandez, R. Bhargava, S.M. Hewitt, I.W. Levin, Nat. Biotechnol. 23, 469 (2005).

[14] C. Petibois, M. Cestelli Guidi, Anal. Bioanal. Chem. 391, 1599 (2008).

[15] C. Petibois, G. Deleris, M. Piccinini, M. Cestelli Guidi, A. Marcelli, Nat. Photon. 3, 179 (2009).

[16] C. Petibois, M. Cestelli Guidi, M. Piccinini, M. Moenner, A. Marcelli, Anal. Bioanal. Chem. 397, 2123 (2010).

[17] C. Petibois, M. Piccinini, M. Cestelli-Guidi, A. Marcelli, J. Synchrotron Rad. 17, 1 (2010).

[18] B. Drogat, M. Bouchecareilh, C. Petibois, G. Déléris, E. Chevet, A. Bikfalvi, M. Moenner, J. Cell Physiol. 212, 463 (2007).

[19] C. Petibois, Anal. Bioanal. Chem. 397, 2051 (2010).

[20] M. Cestelli Guidi, S. Yao, D. Sali, S. Castano, A. Marcelli, C. Petibois, Biotechnol. Adv. 31, 402 (2013). 
[21] C. Petibois, K. Gionnet, M. Goncalves, A. Perromat, M. Moenner, G. Déléris, Analyst 131, 640 (2006).

[22] E. Levenson, P. Lerch, M.C. Martin, Infrared Phys. Technol. 51, 413 (2008).

[23] H. Fabian, P. Lasch, D. Naumann, J. Biomed. Opt. 10, 031103 (2005).

[24] M. Cestelli-Guidi, M. Piccinini, A. Marcelli, A. Nucara, P. Calvani, E. Burattini, J. Opt. Soc. Am. A 22, 2810 (2005).

[25] C. Petibois, B. Desbat, Trends Biotechnol. 28, 495 (2010).

[26] C. Petibois, E. Goormaghtigh, A. Travo, V. Bobroff, Method for determining absorption bands, in: Inserm, France 2014
[27] C. Petibois, Anal. Bioanal. Chem. 397, 2031 (2010).

[28] C. Mayet, A. Deniset-Besseau, R. Prazeres, J.M. Ortega, A. Dazzi, Biotechnol. Adv. 31, 369 (2013).

[29] S. Ghosh, N.A. Kouame, L. Ramos, S. Remita, A. Dazzi, A. Deniset-Besseau, P. Beaunier, F. Goubard, P.H. Aubert, H. Remita, Nat. Mater. 14, 505 (2015).

[30] J. Houel, E. Homeyer, S. Sauvage, P. Boucaud, A. Dazzi, R. Prazeres, J.M. Ortega, Opt. Expr. 17, 10887 (2009).

[31] C. Petibois, Anal. Bioanal. Chem. 401, 783 (2011). 